\title{
COMPARATIVE EVALUATION OF BACT/ALERT 3D CULTURE MEDIUM AND NESTED PCR IN THE DIAGNOSIS OF TUBERCU- LOUS MENINGITIS
}

\author{
Sk Rahaman H, Kumar G, Meena VK, Agarwal A, Nath G, Kumar K \\ Institute of Medical Sciences, Banaras Hindu University, Varanasi - 221005, Uttar Pradesh, India
}

\begin{abstract}
Introduction: We evaluated the efficacy of BacT/ALERT 3D culture medium in comparison with nested polymerase chain reaction (PCR) for early diagnosis of Mycobacterium tuberculosis (MTb) in cerebrospinal fluid (CSF).
\end{abstract}

Methodology: A total of 50 clinically suspected patients with CSF findings suggestive of TBM (Tuberculous meningitis) and 20 healthy controls were included in the present study.CSF from each of the patient was inoculated in BacT/ALERT-MP culture media along with the culture on convention Lowenstein Jensen's medium. Simultaneously nested PCR was carried out both in CSF and growth positive above culture media targeting heat shock protein gene (hsp65), a conserved gene of MTb.

Results: The culture showed 76\% (38/50) positivity in TBM; however, 15\% (3/20) of controls also showed false positivity by BacT/ALERT-MP system. By nested CSF-PCR, TBM could be detected in 92\% (46/50) cases and $5 \%(1 / 20)$ in the controls. The other interesting observation was that when DNA isolated from broth of BacT/ALERT MP culture were subjected for PCR amplification, 15 (39.5\%) of them yielded the desired amplicon for MTb while the broths subjected to conventional $L J$ media yielded for $M$. tuberculosis in 12 of the 38 bottles (31.5\%). None of culture negative medium was positive by PCR. Sensitivity and specificity of BacT/ALERT MP culture considering all of them as real positive were $76 \%$ and $85 \%$ while for nested PCR the corresponding values were $92 \%$ and $95 \%$ respectively.

Conclusions: BacT/ALERT 3D culture medium seems to be unsatisfactory as isolation sensitivity rate is very poor. Moreover, growth indicated in the medium must be confirmed for being Mycobacteria as other contaminants can also grow.

Key words: Mycobacterium tuberculosis, Tuberculous meningitis, Bact/ALERT, CSF PCR.

\section{INTRODUCTION}

WHO has estimated the incidence of new cases of active tuberculosis to be $139 / 100,000$ population annually worldwide with $\sim 1.6$ million deaths per year. ${ }^{1}$ Central nervous system (CNS) tuberculosis is one of the severest forms of the disease resulting into high morbidity, serious neurological defects and mortality. ${ }^{2}$ In the recent past, number of immunocompromised population has increased due to

\author{
Correspondence: \\ Kailash Kumar \\ Professor of General Medicine, New G-15 \\ Aurbindo Colony, BHU campus \\ Varanasi - 221005, UP, India \\ E-mail: kkgbhu@gmail.com
}

high prevalence of HIVIAIDS, increasing incidence of diabetes mellitus, growing geriatric population and increased use of immunosuppressive drugs. Tuberculous meningitis (TBM) is rampant due to above mentioned reasons. ${ }^{1}$ For better prognosis and minimum neurological sequels, an accurate and prompt diagnosis is essential. The diagnosis of TBM, like any other infectious disease, can be made by detecting the whole microorganism and/or its components or by host responses to the invading microorganism. Amongst the direct detection methods; microscopy of smears by differential or special staining is already in practice for Mycobacterium tuberculosis (MTb), but is very poor in sensitivity since number of bacteria in the CSF is usually too low $\left(<10^{4} / \mathrm{ml}\right)$ and similar explanation is also true for poor sensitivity of MTb 
specific antigen detection. ${ }^{2}$ However, cultivation and PCR amplification are the two methods whereby we can increase the number of copies and therefore sensitivity of detection improves. The conventional method of isolation of MTb in CSF is inadequate for early diagnosis as it takes 4-8 weeks apart from being very poor in sensitivity. However, the culture methods have been modified for early detection of the bacterial growth. The BACTEC method yields culture result as early as 7-10 days but has a limitation of disposal of radioactive wastes, incomplete automation and also requires expertise to perform the test. ${ }^{3} \mathrm{BacT} /$ ALERT 3D system, a colorimetric fully automated non-radiometric liquid culture system gives comparable results in relation with isolation of mycobacterium both in terms of sensitivity and time, in comparison to Bactec system. ${ }^{4-7}$ Recently, nucleic acid amplification based assay (NAA), the method of increasing the copy number, has been found as a promising method for early diagnosis of tuberculosis because of its sensitivity, specificity as well as rapidity. ${ }^{8.9}$ Several reports are available showing wide variation $(65-100 \%)$ in ability of detection of MTb in TBM. On the basis of available reports, nested PCR assay has been found to be the most promising. ${ }^{7-16}$ Real time PCR has been reported to be good, but it is less sensitive compared to nested PCR in detection of TBM. ${ }^{16}$ Although, Takahashi et al. ${ }^{16}$ have reported better detection of MTb by combining the real time (RT) PCR with nested PCR assay in CSF. However, later method is technically demanding. Available commercial serological tests provide inconsistent and imprecise results leading to highly variable sensitivity and specificity. Therefore, WHO has strongly recommended that these tests are not to be used for diagnosis of pulmonary and extra pulmonary TB. ${ }^{17}$ The present study therefore was undertaken to evaluate the role of BacT/ALERT 3D culture system as well as nested PCR based detection of MTb for early diagnosis of TBM along with other conventional methods.

\section{METHODOLOGY}

The Present study was carried out in Departments of Medicine, Microbiology and Anesthesiology of the University Hospital of Institute of Medical Sciences, Banaras Hindu University, Varanasi, India during the period of June 2009-May 2011. A total of 50 suspected cases of TBM and 20 healthy controls were included in the study. This study was approved by Institutional Ethics Committee and accordingly written informed consent was taken from each of participants or their attendants.

Case Selection: Patients with a compatible history and clinical/biochemical findings suggestive of TBM were included in the study. Supportive evidences were further collected by carrying out CSF Adenosine deaminase activity (ADA), Monteux test, ESR, Chest X-ray (PA View), CT scan/ MRI of brain. The response to anti-tubercular therapy (ATT) also supported the clinical diagnosis of TBM. Patient with HIV infection or any immune-deficient state, autoimmune disease, viral encephalitis, diabetics, malignancy were excluded from the study.

Controls: CSF was collected from patients undergoing spinal anaesthesia for surgical procedures for traumatic/non-infective condition as healthy controls.

Collection of cerebrospinal fluid: About 3.0-5.0 $\mathrm{ml}$ of CSF was obtained in a sterile vial from each of the patients and controls by performing lumbar puncture aseptically prior to start of ATT. Half of the volume $(1.5-2.5 \mathrm{ml})$ of collected CSF samples were subjected to the following: cytological and biochemical studies, AFB smear examination, BacT/ALERT MP culture immediately without centrifugation while other half was processed later for amplification by PCR for which CSF was preserved at $4^{\circ} \mathrm{C}$.

CSF Culture: Each CSF specimen was inoculated in the BacT/ALERT MP culture media (bioMerieux) by strictly following manufacturer's instructions. In brief: BacT/ALERT MP culture media [18] contains Middle rook $7 \mathrm{H} 9$ broth, pancreatic digests of casein, bovine serum albumin and catalase. BacT/ALERT MP media were brought to room temperature (RT) before inoculation. Disinfection of the top of each media septum was done with an alcohol pad ( $70 \%$ ethanol). Reconstituted antimicrobial agent supplement was added in the volume of $0.5 \mathrm{ml}$ aseptically using a syringe and needle to each of the media. About $0.5 \mathrm{ml}$ each of the CSF sample was inoculated into the appropriately labeled MP media. The inoculated BacT/ALERT MP bottles were incubated in MB 
cabinet. The positive bottles were further subjected to standard Lowenstein Jensen's medium and incubated for 12 weeks for further confirmation by morphological as well as molecular methods.

Extraction of DNA: To extract DNA from the CSF and BacT/ALERT positive culture media, the steps were followed as described by Sambrook et al. ${ }^{19}$

Primers: Heat shock protein gene (hsp65) of MTb is known to be quite conserved and in house designed well tested primers hsp TBF1 and hsp TBR1 were selected for amplification of a 494 bp nucleotide sequence for primary PCR; hspTBF2 and hspTBR2 were selected for amplification of a 218 bp nucleotide sequence for nested PCR. ${ }^{20}$ The oligo sequences (5'-3') were as follows:

\section{For Primary PCR:}

hspTBF1: AAAAGC CGG ATG GCAATT CG hspTBR1: ATT ACC GGC TTG GAC CCC CTG

\section{For Nested PCR:}

hspTBF2: ATG CGG CGC ACA CCG AAG ACA AG

\section{hspTBR2: TCAACG CGC TGT CTA GCT TGT C}

Buffers: Taq polymerase enzymes and customized primers were procured from SBS Genetech Co.,Ltd.,China.

Amplification of DNA: PCR reaction was carried out in $25 \mu \mathrm{l}$ volume. Reaction mix contained $10 \mathrm{x}$ reaction buffer ( $5 \mu /$ sample), dNTPs (concentration $2.5 \mathrm{mmol}$ each base), forward and reverse primers (concentration $10 \mathrm{pmol} / \mu \mathrm{l}$ ) and Taq polymerase enzyme (1U). Amplification was carried out on Biometra, Gottingen Thermo Cycler, Germany with a heated lid. The hot start method was employed by heating at $94^{\circ} \mathrm{C}$ for 5 min initially. Thereafter, amplification was carried out for 35 cycles at $90^{\circ} \mathrm{C}$ for $1 \mathrm{~min}$ (denaturation), $65^{\circ} \mathrm{C}$ for $1 \mathrm{~min}$ (annealing) and $72^{\circ} \mathrm{C}$ for $1 \mathrm{~min}$ (extension). Extra extension was carried out at $72^{\circ} \mathrm{C}$ for $7 \mathrm{~min}$. The amplification products of primary PCR was again amplified with nested primers following same protocol. The final amplification products/bands were analyzed on $2 \%$ agarose gel stained with ethidium bromide under UV light (figure 1). Positive (DNA extracted from M. tuberculosis growth) and negative (double distilled water) controls were run with each batch of samples analyzed. Documentation of gel was done by Multimage ${ }^{T M}$ Light Cabinet, Alpha Innotech Corporation, CA, USA.

Statistical methods: Mean, standard deviations were calculated for quantitative variables. Student Newman Keul Test, $p$-value and f-value have been used to test the significant difference between the mean of two groups. For qualitative and categorical variables, $\mathrm{X}^{2}$ and Z-test have been applied to test the significant difference between two proportions. Sensitivity, specificity, positive and negative predictive value and accuracy were calculated at different cut off points of different variables in respect to PCR.

\section{RESULTS}

None of the CSF specimen was found positive for acid fast bacteria by Ziehl Neelsen staining.

\begin{tabular}{|l|l|l|l|l|}
\hline $\begin{array}{l}\text { Table 1. BacT /ALERT MP culture positivity in } \\
\text { patients with TBM and healthy controls }\end{array}$ \\
\hline & TBM Cases & \multicolumn{2}{l|}{ Controls } \\
\cline { 2 - 6 } & No. & $\%$ & No. & $\%$ \\
\hline BacT/ALERT Test(MP) & & & & \\
\hline Positive & $38^{*}$ & 76 & $3^{* *}$ & 15 \\
\hline Negative & 12 & 24 & 17 & 85 \\
\hline CSF-PCR(nested) & & & & \\
\hline Positive & $46 \#$ & 92 & $1 \# \#$ & 5 \\
\hline Negative & 4 & 8 & 19 & 95 \\
\hline
\end{tabular}

Fisher's Exact Test:

*versus ${ }^{* *} p<0.0001$, \# versus \# $p<0.0001$, versus \#\# $p<0.01$ 
BacT/ALERT MP culture of CSF showed that $76 \%$ of the specimens were positive in TBM while 15\% of controls also yielded for the bacterial growth as indicated by the color change in the medium (table-1). However, detection rates between the two groups were significantly different $(P<0.0001)$. The other interesting observation was that when DNA isolated from broth of BacT/ALERT MP culture was subjected for PCR amplification, 15 $(39.5 \%)$ of them yielded the desired amplicon for $M T b$. Interestingly, when the liquid medium was inoculated to conventional Lowenstein Jensen's medium, 31.5\% (12/38) were found positive for the growth of $M$. tuberculosis while rest of the BacT/ ALERT MP culture media yielded rapid growing contaminants mostly non acid fast bacteria. The PCR based amplification targeting heat shock protein gene (hsp65) of MTb by using nested protocol on CSF of TBM showed positivity in 92\% (46/50) and in healthy controls positive amplification could be observed in $5 \%$ (table 1, figure 1). The detection rate in TBM was significantly higher than the controls $(P<0.0001)$. Further, the detection rate by nested PCR based assay was significantly higher $(p<0.02)$ as compared to BacT/ALERT MP Culture system.

\section{DISCUSSION}

Nucleic acid amplification assays (NAA) have been established as the best technique for rapid and accurate diagnosis of MTb cases in extra pulmonary infection including TBM. Several reports are available showing unsatisfactory sensitivity of detection (32\% to $90 \%)$ of single amplification round which occurs mostly due to low bacterial count as well as presence of PCR inhibitors in biological samples. ${ }^{7-16}$ Although, detection efficiency of real time PCR method is better than the gel electrophoresis, but the performance of this system is also marred by the PCR inhibitors and detection rate ranges between $17.5 \%$ to $93.8 \%{ }^{16}$ The above two methods have usually been found to lack the desired accuracy in diagnosis of TBM. ${ }^{21}$ However, recently introduced nested PCR assay has drastically improved the sensitivity and specificity of diagnosis TBM. The drawback of this system is that increased sensitivity is always associated with risk of contamination. There are several reports based on nested PCR targeting variety of structural and repetitive gene sequence of MTb often showing $100 \%$ sensitivity and specificity. ${ }^{22}$ It has now been proved that nested PCR is 1000 times more sensitive than the conventional single round PCR assay. ${ }^{21}$ Majority of the reports showing better results by nested PCR have targeted MPT 64 gene sequences of MTb. ${ }^{22}$ The MPT 64 genes has been reported to be present exclusively in MTb and not even in BCG strain of Mycobacteria. Although, this makes it specific for MTb, it could not amplify the other members of Mycobacterium complex (M. bovis, $M$. africanus and $M$. microti) which are not rare in TBM. In the present study, we have targeted heat shock protein gene (hsp 65) which is much conserved and primers have been designed in such a way to cover all the four species of Mycobacterium complex. By using in house designed primers, we were able to detect, the Mycobacterium complex in $92 \%$ of CSF specimen of the suspected cases of TBM which is significantly better and faster than the culture method namely BacT/ALERT MP culture system which was found to be positive for the growth in $76 \%$ of the cases. Surprisingly, $5 \%$ of the CSF specimens collected from the healthy (Non tuberculous) individuals were also found positive for desired amplicon. Although, this detection of MTb may be explained on the basis of endemic proportion of tuberculosis in India, the fact that CSF is the second preferred site after lungs for the presence of MTb due to availability of high oxygen concentration and also because of the better detection sensitivity (1- $10 \mathrm{copy} / \mathrm{ml}$ ) of the present assay, which might be able to detect very low copy number of the bacterium. Thus, presence of MTb in CSF of healthy persons cannot be denied in endemic areas. However, it will be interesting to have follow-up of such cases and also further study involving large number of study subjects is essentially needed before drawing any conclusion. Another observation was that DNA isolated from broth of BacT/ALERT MP culture was subjected for PCR amplification, only 12/38 (31.5\%) of them were found positive for the desired amplicon for MTb. One possibility for this observation may be that contaminant bacteria might have grown and this growth might be responsible for color change. Since the rest of the visibly positive bottles were observed with the contaminants growing, the efficacy of this medium becomes doubtful. Further there are reports that many of the saprophytic maycobacteria can also grow in this medium apart from normal commensal flora of the skin 
inadvertently coming into the collection vials. Earlier studies also have shown high contamination rates of $17.1,18.9$, and $11.0 \%$ for BACTEC MGIT 960, ESP II, and Middlebrook agar, respectively when variety of specimens e.g. sputum, urine and stool were submitted for isolation to these automated culture systems. ${ }^{23}$ Saprophytic mycobacteria. M. gordonae was the frequent isolate in these media. ${ }^{23}$ This may lead to unnecessary administration of anti tubercular therapy to the patients if not confirmed by a definite confirmatory method. It may be likely that the inhibitors added to the BacT/ALERT MP culture may not be able to inhibit the contaminants. Presence of PCR inhibitors in this medium seems to be a remote possibility as those CSF specimens who were positive for $M$. tuberculosis complex specific amplification were also positive when PCR was performed from the liquid growth BacT/ALERT MP. Moreover, this observation could further be ratified by growing the $M$. tuberculosis by using conventional LJ medium in almost all the broth with the exception 3 culture broths only.

As all the suspected cases responded to anti tubercular treatment, clinical suspicion was considered as gold standard in the present study. The sensitivity, specificity, positive and negative predictive values of the presented nested PCR assay were $92 \%, 95 \%, 97.8 \%$ and $82.6 \%$ respectively. Although improved isolation is the ideal requirement, our PCR assay seems to be quite satisfactory in detection of Mycobacterium complex in CSF of the suspected cases of TBM. The sensitivity of detection by automated system seems to be disappointing and needs drastic improvement because of high rates contamination added with very high cost of establishment and consumable to run the service in TB diagnostics.

\section{CONCLUSIONS}

Nested PCR based detection of $M$. tuberculosis is definitely better than conventional culture isolation including BacT ALERT system. However, culture isolation has significant role for determination of drug resistance in M. tuberculosis the era of MDR and XDR tuberculosis as PCR based detection system is not available in most of the peripheral laboratories and also unable to detect drug resistance against all the anti tuberculous drugs in use. We have already sdeclared "No to TB serology". The better media for rapid growth are the need of the time. The available BacT ALERT system needs further improvement as observed in the present study since contaminates are also growing. Further, color change of the media should not be considered as an indicator of Mycobacterial growth in this culture system and the growth must be verified before giving the final report. However, study involving large number of patients and controls are warranted on the basis of the present study before drawing any definite conclusion.

\section{REFERENCES}

1. Global tuberculosis control: surveillance, planning, financing. WHO report 2007, Geneva, World Health Organization: WHO/HTM/ TB/ 2007;376.

2. Rock RB, Olin M, Baker CA, Molitor TW, Peterson PK. Central nervous system tuberculosis: pathogenesis and clinical aspects. Clin Microbiol Rev 2008;21:243-61.

3. Harris G, Rayner A, Blair J, Watt B. Comparison of three isolation systems for the culture of mycobacteria from respiratory and non-respiratory samples. J Clin Pathol 2000;53:615-618.

4. Garcia FG, Angulo GP, Garcia FG, et al. Evaluation of the MB/BacT automated mycobacteria culture system versus culture on Lowenstein medium. Clin Microbiol Infect 1998;4:339-43.

5. Palacios JJ, Ferro J, Palma NR. Fully automated liquid culture system compared with LowensteinJensen solid medium for rapid recovery of mycobacteria from clinical samples. Eur J Clin Microbiol Infect Dis 1999;18:265-73.

6. Benjamin WH Jr, Waites KB, Beverly A, et al. Comparison of the MB/BacT system with a revised antibiotic supplement kit to the Bactec 460 system for detection of mycobacteria in clinical specimens. J Clin Microbiol 1998;36:3234

7. Takahashi S, Takahashi T, Kuragano T, et al. A case of chronic renal failure complicated with tuberculous meningitis successfully diagnosed by nested polymerase chain reaction (PCR). Jpn J Nephrol 2005;47:113-20.

8. Liu PYF, ShiZY, Lau YJ, and Hu BS. Rapid diagnosis of tuberculous meningitis by a simplified nested amplification protocol. Neurology 1994;44:161-64. 
9. Scarpellini P, Racca S, Cinque $P$, et al. Nested polymerase chain reaction for diagnosis and monitoring treatment response in AIDS patients with tuberculous meningitis AIDS 1995;9:895-900.

10. Lin HJ, Harn HJ, Hsu WL, Tsao WL, Lee HS, Lee $\mathrm{WH}$. Rapid diagnosis of tuberculous meningitis by polymerase chain reaction assay of cerebrospinal fluid. J Neurol 1995;242:147-52.

11. Wei CY, Lee CN, Chu CH, Hwang JJ and Lee CP. Determination of the sensitivity and specificity of PCR assays using different target DNAs for the detection of Mycobacterium tuberculosis. The Kaohsiung J Med Sci 1999;15:396-405.

12. Caws M, Wilson SM, Clough C, Drobniewski F. Role of IS6110-targeted PCR, culture, biochemical, clinical, and immunological criteria for diagnosis of tuberculous meningitis. $J$ Clin Microbiol 2000;38:150-55.

13. Mehta PK, Raj A, Singh N, Khuller GK. Diagnosis of extrapulmonary tuberculosis by PCR. FEMS Immunol Med Microbiol 2012;66:20-36.

14. Kulkarni $S$, Singh $P$, Memon $A$, et al. An inhouse multiplex PCRtest for the detection of Mycobacterium tuberculosis, its validation \& comparison with a single target. Indian J Med Res 2012;135:788-94.

15. Takahashi T, Nakayama T, Tamura M, et al. Nested polymerase chain reaction for assessing the clinical course of tuberculous meningitis. Neurology 2005;64:1789-793.

16. Takahashi T, Tamura M, Takasu T. The PCR-Based Diagnosis of Central Nervous System Tuberculosis: Up to Date. Tuberculosis Research and Treatment 2012;1155/2012/831292.
17. World Health Organization. Commercial serodiagnostic tests for diagnosis of tuberculosis: policy statement. Geneva, WHO (2011). Available from: http://www.who int/tb/features_ archive/20july11_end_to_inaccurate_tb_blood_ tests/en/index.htmls.

18. Organon -Teknika.2000. BacT/ALERT MB,MP culture media, package inserts. OrganonTeknika, Malvern, $\mathrm{Pa}$.

19. Commonly Used Techniques in Molecular Cloning, Appendix 8 (2001): In Molecular cloning, Vol 3, $3^{\text {rd }}$ ed, by Joseph Sambrook and DW Russell. Cold Spring Harbor Laboratory Press, Cold Spring Harbor, NY, USA.

20. Sareen A, Khare GN, Nath G, Singh S. Role of polymerase chain reaction in osteoarticular tuberculosis. Indian J Ortho 2006;40:264-266.

21. Takahashi T, Tamura M, Asami $Y$ et al. Novel widerange quantitative nested real-time PCR assay for Mycobacterium tuberculosis DNA: clinical application for diagnosis of tuberculous meningitis. J Clin Microbiol 2008;46:1698-707.

22. Rock RB, Olin M, Baker CA, Thomas W. Central nervous system tuberculosis: Pathogenesis and clinical aspects. Clin Microbiol Rev 2008;21:24361.

23. Williams-Bouyer N, Yorke R, Lee HI, Woods GL. Comparison of the BACTEC MGIT 960 and ESP culture system II for growth and detection of mycobacteria. J Clin Microbiol 2000;38:4167-170. 